\title{
Topologies of wireless mesh networks with inband backhauling
}

\author{
Markus Gruber ${ }^{*}$, Siegfried Klein ${ }^{\dagger}$, Serkan Uygungelen ${ }^{\dagger}$, David Chieng $^{\ddagger}$, It Ee Lee ${ }^{\ddagger}$, and Dietrich \\ Zeller $^{\dagger}$
}

\begin{abstract}
Wireless mesh networks (WMNs) with inband backhauling use the same antennas for the backhaul as well as for the access. Therefore antennas of next hop neighbours need to be directed to each other. However, such a configuration is not possible in a three-sectorized hexagonal cell deployment. In this paper we derive several alternative topologies that are suitable for WMNs with inband backhauling. We show that a topology with four directional antennas per node and backhaul connectivity between indirect neighbours outperforms competing topologies in terms of handover rate, optimal maximum power, and system capacity.
\end{abstract}

Index Terms-wireless mesh networks, inband backhauling, network planning, sector shapes

\section{INTRODUCTION}

The demand for data rates in mobile communication systems increases dramatically and telecom network operators have to find ways to accommodate this demand. One strategy is to increase the number of base stations per area because each single base station comes along with additional capacity, which can also be used to optimize interference conditions. However, each added base station also requires a connection to the core network, which is called backhaul. Due to the high cost of wire line backhaul, especially in areas where fixed infrastructure is not widely available, wireless backhaul is an attractive alternative solution and corresponding multihop/mesh concepts are subject to research.

Wireless mesh networks (WMNs) consist of radio nodes that are organized in a mesh topology [1]. In contrast to conventional relays which are clearly attributed to one single neighbouring base station, the mesh nodes we investigate are connected to several neighbours.

In classical cellular networks a hexagonal geometry [2] is applied and access antennas of neighbouring nodes typically do not directly point at each other in order to avoid interference. In WMNs, however, a reliable high throughput connectivity between neighbouring nodes has to be provided. This connectivity can be either out of band, e.g. by a microwave connection using dedicated antennas, or inband

\footnotetext{
* Corresponding Author, email: markus.gruber@alcatel lucent.com

† Alcatel Lucent Bell Labs, Lorenzstr. 10, 70435 Stuttgart, Germany

$\ddagger$ British Telecom Innovate and Design, British Telecommunications Plc Kuala Lumpur, Malaysia
}

using the same antennas for both backhaul and access. Major advantages of inband backhauling are that no extra antennas and thus no additional expenses for equipment are needed and that the operator does not need to possess additional spectrum. Nevertheless, sharing resources between access and backhaul has a substantial impact on the topology. For relays with inband backhauling it has been shown by Balachandran et al. [3] that a significantly better capacity can only be obtained if the antenna beams of nodes that form a backhaul link directly point at each other in order to improve the quality of the backhaul link as much as possible. In this context a threesectorized hexagonal deployment of nodes is not possible anymore because antennas of neighbouring nodes cannot always point at each other. In this work we therefore study several alternative topologies that can be used to support inband backhauling in WMNs. To the best of our knowledge, this work is the first one that analyzes various sector shapes with respect to their suitability to WMNs with inband backhauling.

The rest of the paper is organized as follows: In section II we will derive alternative topologies for WMNs with inband backhauling. Section III displays simulation results showing that a topology with four directional antennas performs better than a topology with six directional antennas as far as the access is concerned. Finally, section IV gives general recommendations for the topology design of WMNs.

\section{COMPARISON OF DIFFERENT TOPOLOGIES}

In this section we discuss different topologies and analyze their corresponding sector shapes. Then, we illustrate why hexagons are not suited for WMNs with inband backhauling and propose alternatives, which are then analytically evaluated with respect to resulting handover rates and energy efficiency. We also introduce a reuse scheme for interference coordination because neighbouring nodes must be able to reach each other and thus must not send on the same resources.

\section{A. Consequences of inband backhauling}

Fig. 1a) lists topological arrangements with different numbers of sectors per node, either with antenna beams of directly neighbouring nodes avoiding each other (avoid) or with antenna beams of directly neighbouring nodes pointing at each other (point). With the term direct neighbours we refer to node pairs that have the shortest possible distance to each 
other, whereas indirect neighbours are those that are farther away.

For typical cellular deployments with three sectors per node, a hexagonal avoid structure is the state-of-the-art topology ${ }^{\S}$. This is the most straightforward arrangement as the hexagonal point topology cannot be arranged with all neighbours pointing at each other. Moreover, in interferencelimited scenarios it is more beneficial to point beams in directions that are not well-covered by other beams rather than wasting energy by competing with other beams.

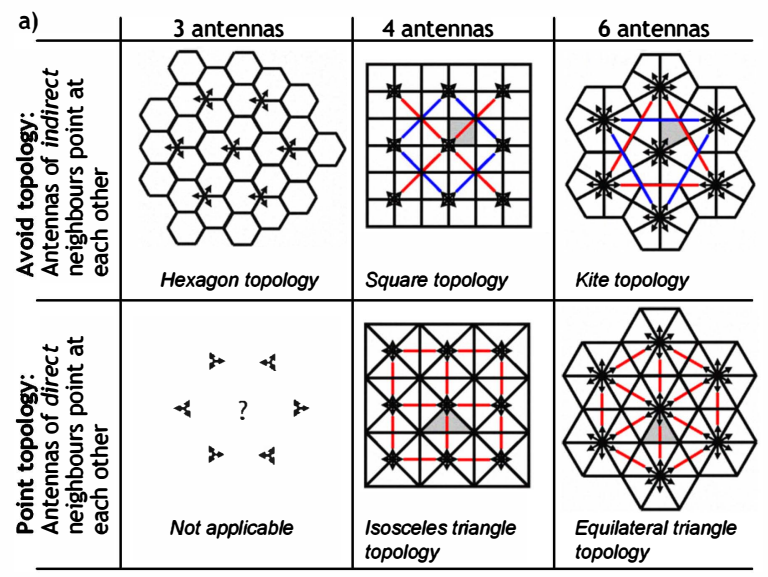

\begin{tabular}{|c|c|c|c|}
\hline b) & $\begin{array}{l}\text { Absolute } \\
\text { perimeter }\end{array}$ & $\begin{array}{l}\text { Relative } \\
\text { perimeter, } \\
\text { relevant for } \\
\text { handover rate }\end{array}$ & $\begin{array}{l}\text { Backhaul link / longe } \\
\text { access link and } \\
\text { corresponding power } \\
\text { w.r.t. } 1 \mathrm{~km} \text { distance }\end{array}$ \\
\hline 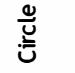 & $3.55 \mathrm{~km}$ & 1 & \\
\hline 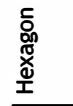 & $3.72 \mathrm{~km}$ & 1.05 & \\
\hline $\begin{array}{l}\frac{\infty}{0} \\
\frac{0}{3}\end{array}$ & $4 \mathrm{~km}$ & 1.13 & $\begin{array}{l}2.83 \mathrm{~km} / 1.41 \mathrm{~km} \\
17 \mathrm{~dB} / 5.6 \mathrm{~dB}\end{array}$ \\
\hline$\stackrel{Ð}{*}$ & $4.15 \mathrm{~km}$ & 1.17 & $\begin{array}{l}4.56 \mathrm{~km} / 1.52 \mathrm{~km} \\
24.8 \mathrm{~dB} / 6.8 \mathrm{~dB}\end{array}$ \\
\hline 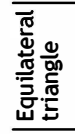 & $4.56 \mathrm{~km}$ & 1.29 & $\begin{array}{l}2.63 \mathrm{~km} / 1.52 \mathrm{~km} \\
15.8 \mathrm{~dB} / 6.8 \mathrm{~dB}\end{array}$ \\
\hline 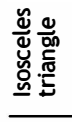 & $4.83 \mathrm{~km}$ & 1.36 & $\begin{array}{l}2 \mathrm{~km} / 1.41 \mathrm{~km} \\
11.3 \mathrm{~dB} / 5.6 \mathrm{~dB}\end{array}$ \\
\hline
\end{tabular}

Fig. 1: a) Topologies with respect to the number of antennas per node and orientation of antenna beams. The resulting shapes represent the access areas served by a sector. Coloured lines represent backhaul connectivity of the mesh network. b) Analysis of sector shapes with propagation model as in Table 1.

Nodes with four antennas [4-6] can be arranged such that all resulting sectors have the same size and shape. The

\footnotetext{
$\S$ It is necessary to have specific antenna characteristics for undistorted hexagons. By contrast, sector shapes for four and six sectorized antennas are independent of the antenna characteristics as long as there is symmetry with respect to the main lobe and equal transmission power for all antennas. This is because these shapes occur in perfectly symmetrical arrangements.
}

corresponding deployment is similar to a chessboard arrangement. Nodes have direct neighbours at distance $d$ and indirect neighbours at distance $d \sqrt{2}$. In the avoid topology, the antennas of direct (i.e. immediate) neighbours avoid each other, but the antennas of indirect neighbours can efficiently reach each other as their antennas perfectly point at each other. In this case sectors have a square shape. Backhaul connectivity between indirect neighbours yields two disjoint sets of nodes (depicted in red and blue) that cannot reach each other with reasonable link quality. This has to be taken into account when deciding which nodes have connectivity to the core network. On the other hand, in the point topology all direct neighbours can reach each other providing full connectivity between all nodes - in this case a sector has the shape of an isosceles triangle.

Sectors with equal size and shape are also obtained when arranging nodes with six antennas. Then there are direct neighbours at distance $d$ ' and indirect neighbours at distance $d^{\prime} \sqrt{3}$. Again, in the avoid topology the backhaul connectivity between indirect neighbours, whose antennas exactly point at each other, results in two disjoint sets (depicted in red and blue). Here the corresponding sector shape is a kite. In the point topology direct neighbours can always reach each other, but in this case the sector shape is an equilateral triangle.

Hence, there are four useful candidate topologies for WMNs: two topologies with four sectors per node (avoid and point) and two topologies with six sectors per node (avoid and point). Fig. 1b) provides an analysis of the corresponding sector shapes with respect to the relative perimeter, the distance of the backhaul link, and the longest access link.

\section{B. Impact of the topology on the handover rate}

The perimeter-to-area-ratio is approximately proportional to the handover rate of a calling terminal since the handover rate is

$H R \approx \frac{E[v] L}{\pi A} \propto \frac{L}{A}$

where $E[v]$ is the expectation value of the terminal speed, $L$ is the perimeter of the sector, and $A$ is the area of the sector [7]. This formula applies to a two-dimensional model where terminals move with random speed $v$ and an independent moving direction randomly distributed in $[0,2 \pi]$. According to this metric, hexagonal topologies have handover rates that are close to the optimum, which is represented by a circle with ideal perimeter-to-area-ratio; however, circles are not relevant for mobile communication systems as they do not tessellate the plane [8].

The perimeter-to-area ratios of shapes in arrangements where nodes of indirect neighbours point at each other, namely the square and the kite, are $8 \%$ and $12 \%$ higher than for hexagons, respectively, and so are the corresponding handover rates. On the other hand, the handover rates imposed by shapes in arrangements where nodes of direct neighbours point at each other, namely the isosceles and equilateral triangle, are even $23 \%$ and $30 \%$ higher than in the hexagonal case. 
In this context it is important to stress that the perimeter of any polygon is additionally influenced by the absolute size of its area as increasing the area by a factor of $n$ comes along with an increase of the perimeter by $\sqrt{n}$. Thus, we always face a tradeoff between few sites with a relatively low number of handovers and many sites with the benefits of spatial reuse occurring whenever additional sites are taken into account. However, this is true for all sector shapes - the differences in handover rates observed above are still valid if a constant size for all sector shapes is assumed. In summary, from a handover perspective avoid topologies perform better than point topologies.

\section{Impact of the topology on energy efficiency}

Now we will look at energy efficiency from both the access and backhaul perspectives. In particular, we will analyze how the maximum transmission powers of different topologies relate to each other when a constant size per sector, exemplarily set to $1 \mathrm{~km}^{2}$, is assumed.

In Fig. 1b) the diameters of the individual sector shapes are listed. This metric addresses the access perspective by asking the question what the farthest distance is that an antenna has to be able to reach for the given area of $1 \mathrm{~km}^{2}$. Again the hexagon has the best diameter $(1.24 \mathrm{~km}$; not mentioned in the figure because it is not applicable for WMNs) followed by shapes that emerge from topologies with four antennas per node, namely the square and the isosceles triangle (both 1.41 $\mathrm{km}$ ). This difference seems to be minor, but the necessary additional power to bridge this gap is by far more, since the path loss increases by almost the fourth power with respect to the distance. When taking the propagation model given in Table 1, we obtain a necessary power increase of about $2.1 \mathrm{~dB}$ to compensate for the above mentioned increase in diameter.

Topologies with six antennas per node, referring to the kite and the equilateral triangle as sector shapes, have an even higher diameter, namely $1.52 \mathrm{~km}$. This requires about $1.2 \mathrm{~dB}$ more power than in topologies with four antennas per node and a diameter of $1.41 \mathrm{~km}$.

As far as the backhaul is concerned, the distances between base stations whose antenna beams point at each other is significantly larger than for the access considerations, so the distances that have to be bridged vary from $2 \mathrm{~km}$ in the case of the isosceles triangle topology to $4.56 \mathrm{~km}$ in the kite topology. Accordingly, the additional power that is necessary with respect to a distance of $1 \mathrm{~km}$ varies between $11.3 \mathrm{~dB}$ and 24.8 $\mathrm{dB}$ based on the propagation model in Table 1, whereas the corresponding powers in the access vary between $5.6 \mathrm{~dB}$ and $6.8 \mathrm{~dB}$ (Fig. 1b).

Thus, at first sight the backhaul requires more power than the access. However, in contrast to a mobile station, a neighbouring base station is usually attached to some higher point and is more exposed. This height gain can be estimated e.g. by using the Hata model; accordingly, with a height gain of $\sim 11 \mathrm{~dB}(5 \mathrm{~dB})$ for a height of $15 \mathrm{~m}(5 \mathrm{~m})$ and the directional antenna gain contributing another $\sim 15 \mathrm{~dB}$, the maximum power that is necessary for the access is always higher than that for the backhaul.

In conclusion, in order to cover a given area with an antenna, sector shapes emerging from topologies with four antennas per node require less maximum power than shapes emerging from topologies with six antennas per node. Please note that this analysis only addresses one isolated aspect of energy efficiency and does not take into account benefits that arise when multiple sectors are served from one single base station. For example, the fact that infrastructure elements as the cooling system are only necessary once per node is not considered here. However, the sector geometry does contribute to the overall energy balance and has to our knowledge not been analyzed for WMNs before.

\section{Mesh node coordination for interference control}

In WMNs with inband backhauling, neighbouring nodes must use separate resources in order to be able to reach each other. For the point topologies this problem is exemplarily solved by employing a resource reuse of 4 . This is depicted in Fig. 2 where only nodes of the same colour may send on the same resources. With this reuse scheme, the area covered by a base station on its assigned resources is four times as large as before. However, as base stations sending on the same resource are evenly distributed, the nature of the resulting coverage shapes remains the same. In other words, we have a perfect cell split.
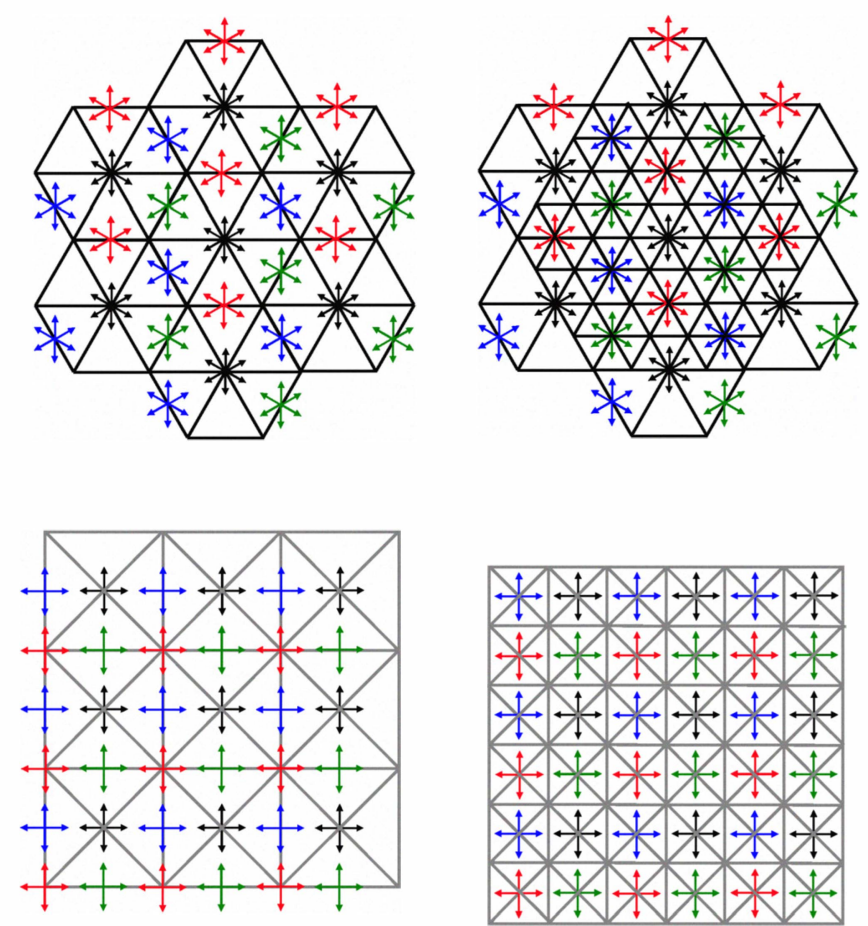

Figure 2: Resource reuse of 4 for the point topologies with six (top) and four (bottom) antennas. Different resources are represented by different colours. Left side: sectors shaped by the black nodes only (disregarding otherwise coloured nodes); right side: sectors that are eventually served by the ensemble of all nodes

The reuse schemes for the point topologies as depicted in Fig. 2 are equally applicable to the avoid topologies. In this case all antenna directions are turned by $45^{\circ}$ and $30^{\circ}$ for four and six antennas per node, respectively. This is possible because the reuse schemes provide different colours not only for direct neighbours, but also for indirect neighbours. For symmetry reasons, we again have a perfect cell split. 
Indirect neighbours are certainly farther away from each other than direct neighbours, but this does not have any negative effect on the backhaul channel capacity. This is because the reuse scheme is chosen such that interference contributions from neighbouring nodes are maximally attenuated thanks to the antenna characteristics.

The reuse schemes are also helpful because a tessellation with isosceles triangles, for instance, comes along with spots that are reached by 8 antennas with equal signal intensity with a reuse scheme these spots occur at different locations on different resources so that schedulers can completely avoid them. This may require a certain degree of cooperation between schedulers of neighbouring nodes though.

The fact that all sectors of the same node use the same resources comes along with high intra-cell interference. However, this cannot be avoided as otherwise one sector antenna can be in transmission mode while a neighbouring sector antenna is in reception mode.

In this study, we do not focus on the tradeoff between the additional resources necessary for a reuse scheme and the gain one gets thanks to the avoidance of interference, but we rather investigate the differences between the sector shapes that emerge in different topologies.

\section{SYSTEM SIMULATION}

We compared the terminal signal to interference and noise ratios (SINRs) of the candidate shapes for WMNs with inband backhauling by means of a system simulator using two dimensional antennas. Considering the resource reuse of four, we took a fourth of the area of a sector of a base station with three access antennas and the typically assumed inter-site distance of $500 \mathrm{~m}$ for urban environments as the reference area of $0.018 \mathrm{~km}^{2}$ in order to ensure a fair comparison among the different shapes. Basic simulation parameters were selected from the recommendations in 3GPP technical report 30.03 [10], with the following modifications to take into account the different nature of the new sector shapes:

- The $3 \mathrm{~dB}$ horizontal antenna beam width of $70^{\circ}[10]$ is multiplied by $3 / 4(3 / 6)$ for four (six) antennas in order to accommodate the reduced opening angles.

- The base station maximum transmission gain of 15 $\mathrm{dB}[10]$ is multiplied by $4 / 3(6 / 3)$ resulting in $16 \mathrm{~dB}$ $(18 \mathrm{~dB})$ for four (six) antennas per node.

- The inter-site distance is adapted to $269 \mathrm{~m}$ and $354 \mathrm{~m}$ for four and six antennas per node, respectively, in order to comply with the fixed area size per sector of $0.018 \mathrm{~km}^{2}$.

As for the inter-site distance, there are two options to ensure a fair comparison: One is to keep the inter-site distance constant so that the number of sites per area remains the same in all cases, but then nodes with a larger number of antennas will always be able to provide a higher capacity than nodes with a lower number of antennas. As we were more interested in the shape of the sectors, we kept the number of sectors per area constant, regardless of the underlying shape, in order to distill the inherent properties of the shapes and the corresponding topologies.

For topologies with four sectors per node, we considered a simulation area where nodes of various colours had an inter- site distance of $269 \mathrm{~m}$, but where nodes of the same colour had twice this inter-site distance, namely $538 \mathrm{~m}$. Likewise, for topologies with six sectors per node, the inter-site distance of $354 \mathrm{~m}$ for various colours rises to $708 \mathrm{~m}$ for nodes of the same colour. Then we analyzed the sectors of a given node and took into account the interference of the first two tiers of nodes of the same colour.

Mobiles were randomly dropped in the simulation area and performed a random walk. The signals of all sectors were measured at the mobiles and mobiles were associated to the sector with the strongest signal. After this mapping had been defined, the SINR was calculated for the current position of the mobiles. For each simulated network shape, global distribution statistics of the SINR values were observed. Then the channel capacity of a user was calculated by using the Shannon-Hartley theorem [11]. Correspondingly, the capacity of a sector was calculated by summing up the channel capacities of all its associated users.

\begin{tabular}{|l|l|}
\hline Parameter & Assumption \\
\hline Cell Type & $\{4,6\}$-sector shapes \\
\hline Inter-site distance & $\{269 \mathrm{~m}, 354 \mathrm{~m}\}$ \\
\hline Area of a sector & $0.018 \mathrm{~km}^{2}$ \\
\hline Propagation Model & $128.1+37.6^{*} \log 10(\mathrm{~d})$ (Distance $\mathrm{d}$ in $\left.\mathrm{km}\right)$ \\
\hline $\begin{array}{l}\text { Minimum distance between terminal and } \\
\text { base station }\end{array}$ & $35 \mathrm{~m}$ \\
\hline Carrier Frequency & $2 \mathrm{GHz}$ \\
\hline System Bandwidth & $10 \mathrm{MHz}$ \\
\hline Thermal noise & $-104 \mathrm{dBm}$ at $10 \mathrm{MHz}$ bandwidth \\
\hline Base station transmission power & $43 \mathrm{dBm}$ \\
\hline Base station antenna horizontal pattern & $\left\{52.5^{\circ}, 359\right.$, dB beam width \\
$20 \mathrm{~dB}$ backward attenuation \\
\hline $\begin{array}{l}\text { Base station antenna maximum } \\
\text { transmission gain }\end{array}$ & $\{16 \mathrm{dBi}, 18 \mathrm{dBi}\}$ \\
\hline Number of terminals & 15 per sector in average (Poisson distribution) \\
\hline Terminal reception noise figure & $9 \mathrm{~dB}$ \\
\hline Scheduling & Resource fair with respect to bandwidth \\
\hline Buffer status at base stations & Full buffer \\
\hline
\end{tabular}

Table 1: Simulation parameters. Whenever numbers vary for different shapes, a set $\{x, y\}$ is given for 4 and 6 sectors.

In order to reduce the complexity of the simulations, shadow fading was disabled. The simulator was constructed based on simulation libraries by Alcatel-Lucent Bell Labs and the University of Stuttgart, Germany. Table 1 summarizes the main parameters of the simulation model.

The results of the simulation are shown in Fig. 3. The four candidate topologies for WMNs with inband backhauling, namely the ones with four and six antennas per node shown in Fig. 1, are investigated. The topologies are evaluated in terms of terminal SINR, user capacity, and sector capacity.

One observation is that for a given number of antennas per node, the avoid topology performs better than the point topology. The avoid topology with four sectors per node performs best, whereas the point topology with six sectors per node performs worst for most quantiles. For the two remaining topologies the picture is less clear: Both the point topology with four sectors and the avoid topology with six sectors display moderate performance results. The former outperforms the latter for the best users in terms of SINR, whereas the situation is the other way around for the worst users. 
a)

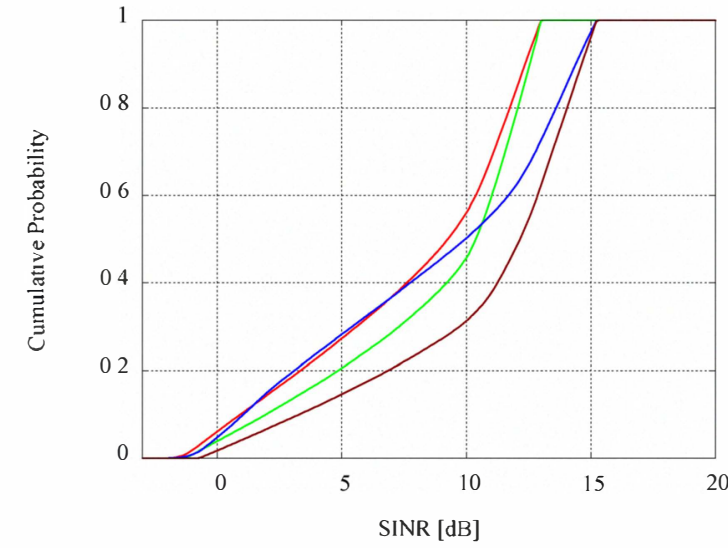

b)

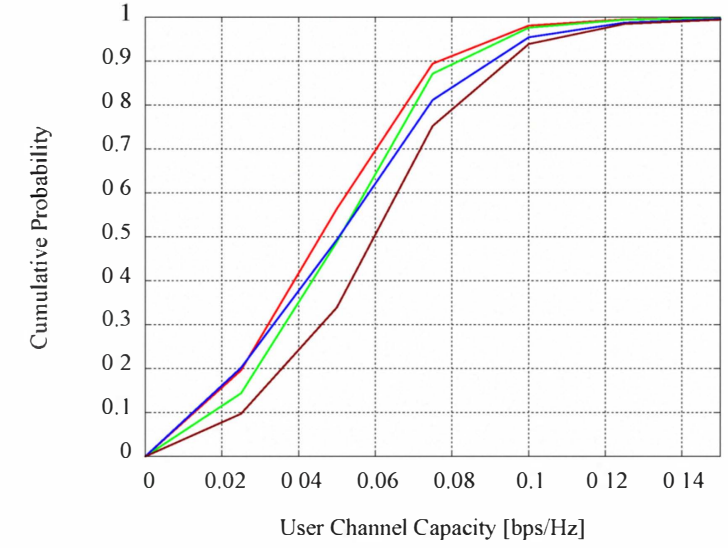

c)

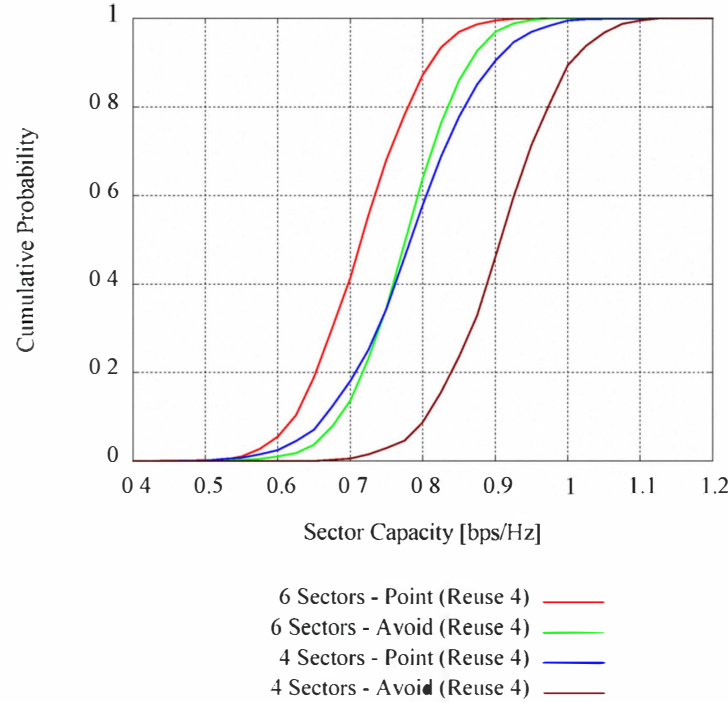

Figure 3: Cumulative probability density functions for a) the terminal SINR, b) the user channel capacity, and c) the sector capacity for the four candidate shapes.

These results have a substantial impact on the network planning of WMNs, at least for networks that are set up from scratch, because sites with four sectors are arranged in a chessboard-like grid, whereas sites with six sectors are arranged in a hexagonal grid (Fig. 2).
At the same time, one has of course to keep in mind that no real deployment today comes along with perfect geometrical shapes - shadowing and reflections induce distortions of the shapes and usually it is not possible to place a base station at the theoretically perfect location. On the other hand, these theoretical considerations are only the starting point of network planning. It can be fatal to begin with an unfavourable topology whose flaws propagate throughout later stages of the planning process.

\section{CONCLUSION AND FUTURE WORK}

In WMNs with inband backhauling, antennas of directly or indirectly neighbouring nodes should point at each other in order to ensure optimal backhaul quality. This, however, cannot be realized with equally sized hexagons. Therefore we have proposed alternative topologies with four and six antennas per node. In this context, we investigated two types of conformations, one where direct neighbours had their antennas point at each other (the point topology) and one where antennas of directly neighbouring nodes avoided each other with the result that antennas of indirect neighbours directly pointed at each other (the avoid topology).

We were able to show that the avoid topology with four antennas per base station and square-shaped sectors outperforms competing topologies in all investigated disciplines, namely handover rate, optimal maximum power (energy efficiency), and system performance in terms of terminal SINR, user capacity, and sector capacity.

To the best of our knowledge this study is the first one that analyzes various sector shapes with respect to their suitability to WMNs with inband backhauling. The findings we provide here may imply a new topology paradigm for greenfield WMN deployments that fundamentally differs from the hexagonal cell structure known today.

For further investigations providing even deeper insights, it would be desirable to extend the two dimensional antenna model used in this paper to a three dimensional model taking into account aspects as the vertical antenna tilt and adaptive array antennas. Our results are based on a radio access simulation supported by some qualitative backhaul analysis so future work should aim to further support these results by a more rigorous backhaul analysis. Then the backhaul SINR can be used to determine the ratio of access resources to backhaul resources in each sector such that the amount of access resources is fairly distributed over all sectors depending on the number and locations of nodes with connectivity to the core network.

\section{ACKNOWLEDGMENT}

The research leading to these results has received funding from the European Community's Seventh Framework Programme (FP7/2007-2013) under grant agreement $\mathrm{n}^{\circ}$ 214994. We would also like to thank Oliver Blume from Alcatel-Lucent Bell Labs and Christian Müller from University of Stuttgart for helpful discussions.

\section{REFERENCES}

[1] I. F. Akyildiz and X. Wang, "A survey on wireless mesh networks", IEEE Communications Magazine, vol. 43 (9), pp. 23 30, 2005 
[2] V. H. Mac Donald, "The cellular concept", The Bell System Technical Journal, vol. 58, pp. 15 41, 1979

[3] K. Balachandran, J. Kang, K. Karakayali, and J. Singh, "Capacity benefits of relays with in band backhauling in cellular networks", IEEE International Conference on Communications, 2008

[4] L C. Wang and K. K. Leung, "Performance enhancement by narrow beam quad sector cell and interleaved channel assignment in wireless networks", Global Telecommunications Conference, vol. 5, 2719 2724, 1999

[5] L. C. Wang and K. K. Leung, "A high capacity wireless network by quad sector cell and interleaved channel assignment", IEEE Journal on Selected Areas in Communications, vol. 18 (3), 472 480, 2000

[6] O. W. Ata, H. Seki, and A. Paulraj, "Capacity enhancement in quad sector cell architecture with interleaved channel and polarization assignments, IEEE International Conference on Communications, vol. 7, 2317 2321, 2001

[7] H. Xie and S. Kuek, "Priority handoff analysis", IEEE Vehicular Technology Conference, 855 858, 1993

[8] F. Aurenhammer, "Voronoi diagrams a survey of a fundamental geometric data structure", ACM Computing Surveys (CSUR), vol. 23 (3), 345 405, 1991

[9] Further enhancement for E UTRA physical layer aspects (release 9), 3GPP TSG RAN TR 36.814 V0.4.1

[10] Universal Mobile Telecommunications System (UMTS); Selection procedures for the choice of radio transmission technologies of the UMTS (UMTS 30.03 version 3.2.0)

[11] R. V. L. Hartley, "Transmission of information", Bell System Technical Journal, 1928 